\section{Fatal acute necrotizing pancreatitis in a 15 years old boy, is it multisystem inflammatory syndrome in children associated with COVID-19; MIS-C?}

\author{
Masoumeh Asgarshirazi ${ }^{1 *}$, Khadije Daneshjou², Seyed Reza \\ Raeeskarami ${ }^{3}$, Mohammad Reza Keramati ${ }^{4}$ and Samrand \\ Fattah Ghazi ${ }^{5}$
}

\begin{abstract}
${ }^{1}$ Associate Professor of Pediatric Gastroenterology, Department of Pediatrics, Imam Khomeini Hospital Complex, Tehran University of Medical Sciences. Tehran, Iran ${ }^{2}$ Full Professor of Pediatric Infectious Diseases, Department of Pediatrics, Imam Khomeini Hospital Complex, Tehran University of Medical Sciences. Tehran, Iran ${ }^{3}$ Associate Professor of Pediatric Rheumatology, Department of Pediatrics, Imam Khomeini Hospital Complex, Tehran University of Medical Sciences. Tehran, Iran ${ }^{4}$ Associate Professor of Surgery, Department of Surgery, Imam Khomeini Hospital Complex, Tehran University of Medical Sciences. Tehran, Iran ${ }^{5}$ Assistant Professor of Critical Care and Anesthesiology, Department of Anesthesiology, Imam Khomeini Hospital Complex, Tehran University of Medical Sciences. Tehran, Iran
\end{abstract}

\section{More Information}

*Address for Correspondence: Masoumeh Asgarshirazi, MD, Associate Professor of Pediatric Gastroenterology, Department of Pediatrics, Imam Khomeini Hospital Complex, Tehran University of Medical Sciences, Tehran Iran, Email: dr.m.asgarshirazi@gmail.com

Submitted: November 16, 2021

Approved: January 12, 2022

Published: January 13, 2022

How to cite this article: Asgarshirazi $\mathrm{M}$ Daneshjou K, Raeeskarami SR, Keramati MR, Ghazi SF. Fatal acute necrotizing pancreatitis in a 15 years old boy, is it multisystem inflammatory syndrome in children associated with COVID-19; MIS-C? Arch Case Rep. 2022; 6: 001-004.

DOI: 10.29328/journal.acr.1001056

Copyright License: () 2022 Asgarshirazi M, et al. This is an open access article distributed under the Creative Commons Attribution License, which permits unrestricted use, distribution, and reproduction in any medium, provided the original work is properly cited.

Keywords: Acute necrotizing pancreatitis; MIS-C; COVID-19

W) Check for updates

OPEN ACCESS

\title{
Abstract
}

Acute pancreatitis in childhood is not common and viral and bacterial infections, bile duct diseases, medications, systemic diseases, trauma, metabolic diseases, and hyperlipidemia are among the most common causes in them. Acute necrotizing pancreatitis is even rarer.

The clinical presentation of Multisystem Inflammatory Syndrome in Children associated with COVID-19 (MIS-C) includes fever, severe illness, and the involvement of two or more organ systems, in combination with laboratory evidence of inflammation and with or without laboratory or epidemiologic evidence of SARS-CoV-2 infection. We present a case of a 15 years old boy with fatal acute necrotizing pancreatitis that fulfilled MIS-C definition based on RCPCH (Royal College of Pediatrics and Child Health) and CPSP (Canadian Pediatric Surveillance Program) criteria.

\section{Introduction}

Acute pancreatitis should be diagnosed if the patient presents with at least two of the following three criteria: abdominal pain, serum amylase and lipase levels higher than three times the normal range, and imaging findings indicating inflammation and involvement of the pancreas [1]. Necrotizing pancreatitis (NP) represents the most severe form of acute pancreatitis with higher mortality and morbidity [2].

Coronavirus disease 2019 (COVID-19) is usually mild in children and adolescents and is asymptomatic in $16 \%$ of cases. It has been reported that $22 \%$ of infected cases have gastrointestinal symptoms. The most common gastrointestinal symptom is vomiting or diarrhea in $17 \%$ of children [1]. There are case reports and retrospective studies of acute pancreatitis associated with COVID-19 [3-10].

In the latter half of April 2020, a novel syndrome in children and adolescents termed multisystem inflammatory syndrome in children (MIS-C) with likely relation to SARSCoV-2 infection was first described. Preliminary accounts of the features of this syndrome resemble those of known entities such as Kawasaki Disease (KD), toxic shock syndrome (TSS), and secondary hemophagocytic lymphohistiocytosis (SHLH)/macrophage activation syndrome (MAS) [11].

\section{Case report}

We present a 15 years old cerebral palsy (CP) boy with severe abdominal pain which began oneweek before admission. 
He was febrile $\left(\mathrm{T}=39^{\circ} \mathrm{c}\right)$, did not have any vomiting and was able to communicate to reveal epigastric tenderness. His past medical history was negative for any trauma, medication or predisposing condition for pancreatitis. He had diarrhea from two days before admission too. Early investigations showed high serum Amylase and Lipase, and pancreas necrosis with peripancreatic fluid collection (fulfilling all three criteria of acute pancreatitis as per the revised Atlanta classification) and some mesenteric lymphadenopathy and terminal ileum thickening in IV and oral contrast enhanced Abdominopelvic CT Scan (Figure 1).

Surgeon visited him and discarded surgical intervention and suggested conservative management. He was ill but stable in vital signs. First Complete Blood Count showed lymphopenia and very high CRP and ERS so we checked inflammatory markers of procalcitonin, D-Dimer, fibrinogen, pro BNP and ferritin in addition to serum electrolytes and biochemistry.

Most of his inflammatory markers became positive. Systemic Inflammatory Response Syndrome(SIRS) can be seen in severe pancreatitis, but his multiple organ involvement including of hematuria, diarrhea and red chapped lips made us think of Multisystem Inflammatory Syndrome in Children (MIS-C) associated with COVID-19. Very high ProCalcitonin, high Fibrinogen, high Pro-BNP(brain natriuretic peptide) and high Troponin I also supported this probable diagnosis. His parents did not give any history of contact or symptoms of COVID-19. Pediatric cardiology consultation was done and ejection fraction and coronary arteries were reported normal. He fulfilled RCPCH (Royal College of Pediatrics and Child Health) and CPSP (Canadian Pediatric Surveillance Program) criteria for MIS-C but his nasopharyngeal SARS-CoV-2 PCR and serology for COVID-19 IgG was negative [12]. Considering the clinical and imaging findings and based on NASPGHAN (North American Society For Pediatric Gastroenterology, Hepatology \& Nutrition) criteria he has moderately severe pancreatitis as for local necrosis and complication [13].
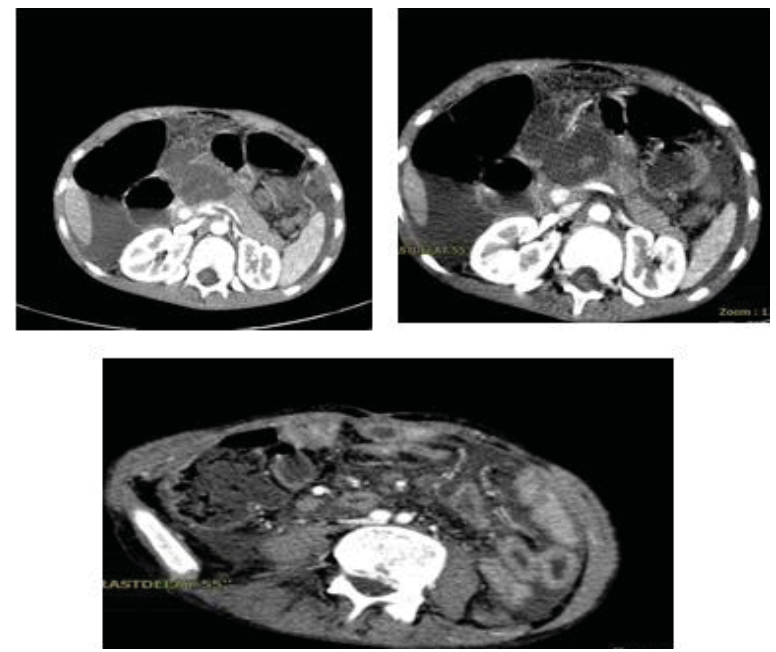

Figure 1: First Abdominopelvic CT scan with IV and oral contrast at admission.
Conservative treatment and monitoring was done and after sampling for blood culture and urine culture antibiotic coverage for gram-negative pathogens and enterococci was prescribed (vancomycin and cefepime). Pediatric infectious disease and pediatric rheumatology consultation was done and intravenous immunoglobulin (IVIG) in dose of 1 gram per $\mathrm{kg}$ of weight $(\mathrm{gr} / \mathrm{kg}$ ) and methylprednisolone pulse therapy (30 $\mathrm{mg} / \mathrm{kg}$ up to $1 \mathrm{gr}$ ) for 3 day was prescribed based on probable diagnosis of MIS-C.[11] Consultants also recommended Aspirin in anti-inflammatory and then antiplatelet dosage and oral prednisolone in dose of $1 \mathrm{mg} /$ $\mathrm{kg} /$ day but patient's condition was not suitable for oral drugs so we used equivalent intravenous Hydrocortisone and subcutaneous daily Enoxaparin. We repeated the abdominopelvic CT scan with IV contrast on sixth day of admission based on surgical consultation since he was still febrile and complained from abdominal pain (Figure 2) and exploratory laparotomy and irrigation and drainage was done and repeated for another four times [13].

His laboratory data is shown in Table 1.

He was intubated and undergone assisted ventilation after third surgery. The first time's culture from intra-abdominal fluid were negative but third sampling on $20^{\text {th }}$ day of admission was positive for Acinetobacter and sensitive to Colistin so after repeated infectious disease consultation, previous antibiotics were discontinued and colistin and Amikacin prescribed. He showed anemia after one week, thrombocytopenia after 12 days and leukopenia after 16 days of admission. His retic count was low and pediatrics hematology consultation and bone marrow aspiration (BMA) for HLH or MAS investigation was done which was reported hypocellular bone marrow without any sign of macrophage phagocytosis. After 3 weeks of admission, he was respirator dependent so a lung CT scan was performed and was reported compatible with COVID-19 involvement or ARDS (acute respiratory distress syndrome) (Figure 3). Repeated SARS-CoV-2 PCR was negative.
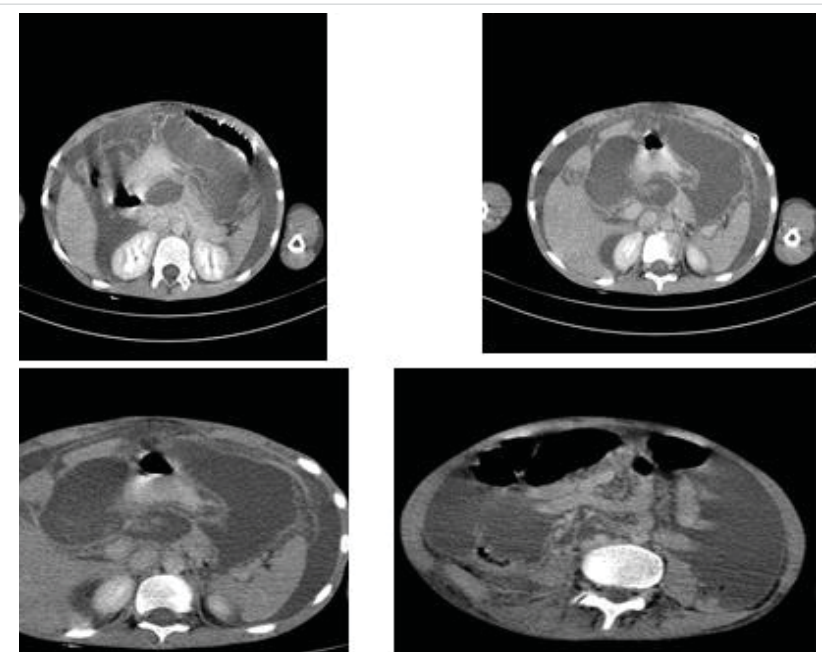

Figure 2: Abdominopelvic CT scan with IV contrast, $6^{\text {th }}$ day of hospitalization. 


\begin{tabular}{|c|c|c|c|c|c|c|c|c|}
\hline $\begin{array}{l}\text { Day of } \\
\text { admission } \\
\text { lab data }\end{array}$ & 1 & 3 & 7 & 10 & 12 & 14 & 16 & 20 \\
\hline $\begin{array}{l}\text { CBC; } \\
\text { WBC } \\
\text { Lymph\% } \\
\text { Neutrophil\% } \\
\text { Hb(gr/dl) } \\
\text { Platelets }\end{array}$ & $\begin{array}{l}15,900 \\
6.3 \\
89.6 \\
14.7 \\
527,000\end{array}$ & $\begin{array}{l}16,400 \\
4 \\
93.1 \\
11.2 \\
364,000\end{array}$ & $\begin{array}{l}14,500 \\
2.8 \\
93.6 \\
9 \\
238,000\end{array}$ & $\begin{array}{l}11,100 \\
6.9 \\
86.3 \\
8.8 \\
247,000\end{array}$ & $\begin{array}{l}9,900 \\
6.4 \\
89.8 \\
8.9 \\
79,000\end{array}$ & $\begin{array}{l}5,900 \\
8.5 \\
89.6 \\
7.6 \\
67,000\end{array}$ & $\begin{array}{l}2,300 \\
7 \\
42,000\end{array}$ & $\begin{array}{l}2,100 \\
19.8 \\
77 \\
6.2 \\
36000\end{array}$ \\
\hline Amylase(serum)(nl<100) & 400 & 324 & 221 & & 647 & & 36 & \\
\hline 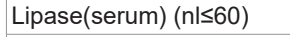 & 357 & & & & & & 18 & \\
\hline AST & 43 & & 45 & & 33 & & 45 & 35 \\
\hline ALT & 12 & & 25 & & 46 & & 27 & 39 \\
\hline $\begin{array}{l}\text { Bili; } \\
\text { Total } \\
\text { Direct }\end{array}$ & $\begin{array}{l}0.8 \\
0.4\end{array}$ & & $\begin{array}{l}0.6 \\
0.3\end{array}$ & & $\begin{array}{l}0.9 \\
0.3\end{array}$ & & & $\begin{array}{l}0.8 \\
0.3\end{array}$ \\
\hline Albumin(gr/dl) & 3 & 3 & 2.5 & 2.4 & 2.8 & & 2.2 & 2 \\
\hline $\begin{array}{l}\text { Pt s, ( INR) } \\
\text { Ptt s }\end{array}$ & $\begin{array}{l}16.8,(1.37) \\
25\end{array}$ & $\begin{array}{l}16.1,(1.32) \\
25\end{array}$ & & $\begin{array}{l}19.9,(1.56) \\
36\end{array}$ & & & & $\begin{array}{l}16.8(1.3) \\
34\end{array}$ \\
\hline Calcium(serum) & 8.9 & 8.8 & 6.9 & 7.3 & 7 & 7.8 & 8 & 6.7 \\
\hline LDH $(n \mid<480)$ & 1145 & & & & 400 & & & \\
\hline Fibrinogen(nl:200-400) & 485 & & & & & & 169 & \\
\hline CPK $(n \mid<370)$ & 357 & & & & & & & \\
\hline CPK-MB & 38 & & & & & & & \\
\hline Troponin I $(\mathrm{n} \mid<0.034)$ & 0.014 & 0.047 & & & & & & \\
\hline Ferritin(nl:10-160) ng/ml & 328 & & & & 647 & & & \\
\hline ProBNP $(\mathrm{nl}<125)$ & 759.02 & 1161 & & & & & 15 & \\
\hline D-Dimer $(n \mid<0.55)$ & 19.48 & & 9.8 & & & & 4.55 & \\
\hline $\begin{array}{l}\text { Pro-calcitonin }(\mathrm{nl}<0.1) \\
\mathrm{ng} / \mathrm{ml}\end{array}$ & 9.99 & & $>30$ & & & & & \\
\hline $\begin{array}{l}\text { U/A; } \\
\text { SG } \\
\text { Protein } \\
\text { Blood } \\
\text { RBC } \\
\text { WBC }\end{array}$ & $\begin{array}{l}1.015 \\
\text { Trace } \\
\text { Trace } \\
10-15 \\
4-5\end{array}$ & & $\begin{array}{l}1.020 \\
\text { Trace } \\
4+ \\
\text { Many } \\
6-8\end{array}$ & & & $\begin{array}{l}1.020 \\
\text { Trace } \\
4+ \\
\text { Many } \\
4-5\end{array}$ & & \\
\hline \begin{tabular}{l} 
Intra abdominal fluid; \\
Appearance \\
Bilirubin T \\
\multicolumn{1}{c}{ D } \\
Albumin \\
Protein \\
LDH \\
WBC (L\%) \\
$\quad$ (PMN\%) \\
RBC \\
Glucose \\
Amylase \\
Lipase \\
Culture
\end{tabular} & & & $\begin{array}{l}\text { Semiturbid } \\
\quad 3.7 \\
\quad 1.1 \\
770 \mathrm{mg} \\
2500 \mathrm{mg} \\
1608 \\
240(59 \%) \\
\quad(41 \%) \\
10 \\
94 \\
2989 \\
1960 \\
\text { Negative }\end{array}$ & & & & & $\begin{array}{l}\text { Acinetobacter } \\
\text { Sensitive to Colistin }\end{array}$ \\
\hline
\end{tabular}
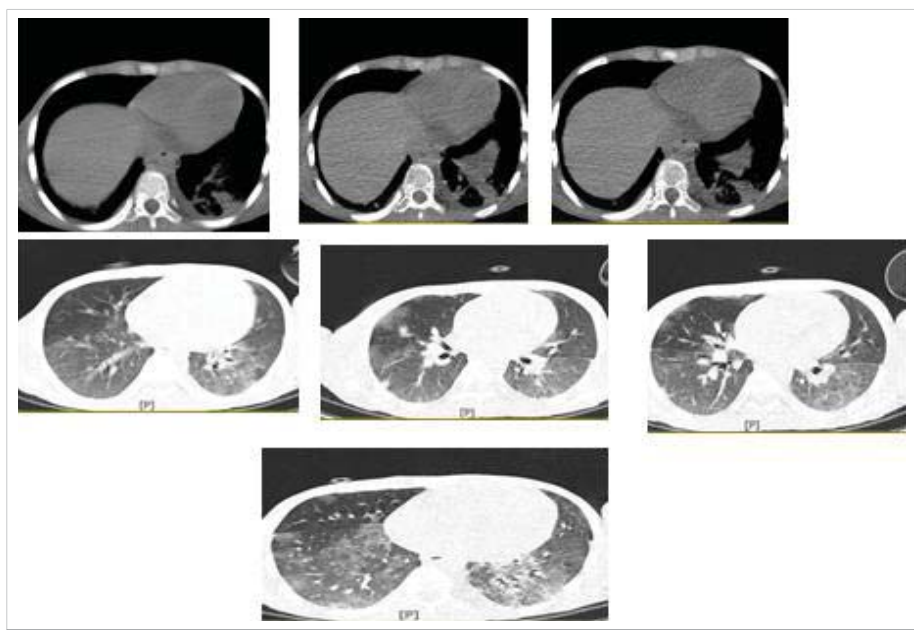

Figure 3: Lung CT scan with and without contrast at $20^{\text {th }}$ day of hospitalization.
Based on repeated pediatric infectious disease consultation on the $21^{\text {st }}$ day of admission intravenous Remdesivir in dose of $5 \mathrm{mg} / \mathrm{kg}$ for first day and $2.5 \mathrm{mg} / \mathrm{kg}$ for other four days was begun. Unfortunately, our patient expired after about one month hospitalization.

\section{Discussion}

Acute pancreatitis should be diagnosed if the patient presents with at least two of the following three criteria: abdominal pain, serum amylase and lipase levels higher than three times the normal range, and imaging findings indicating inflammation and involvement of the pancreas [1]. Viral and bacterial infections, bile duct diseases, medications, systemic diseases, trauma, metabolic diseases, and hyperlipidemia 
are the most common causes of pancreatitis in childhood. Necrotizing pancreatitis is the most severe form of acute pancreatitis and is associated with a poor prognosis $[2,8]$.

COVID-19 infection, caused by SARS-CoV-2, mainly causes pulmonary disease but involvement of gastrointestinal and hepatobiliary systems, cardiac, and renal systems and vascular events has been reported $[6,10]$. The mechanism for the development of pancreatitis is unclear; perhaps due to a direct cytopathic effect from the COVID-19 virus, as has been implicated in other viral causes of pediatric pancreatitis $[6,8]$. In adult patients with severe COVID-19, a strong inflammatory response consistent with cytokine storm has been reported. The cytokine storm plays a critical role in the development of acute respiratory distress syndrome, systemic inflammatory response, and multi-organ failure in adults. On April 27, 2020, the United Kingdom reported a cluster of eight severely ill pediatric cases displaying a phenotype that resembled a combined state of incomplete Kawasaki Disease (KD) and Toxic Shock Syndrome (TSS), including signs of circulatory shock and an overall hyperinflammatory state which were different from the inflammatory response in adults based on time and some clinical and biochemical properties. All children tested negative for SARS-CoV-2 on bronchoalveolar lavage or nasopharyngeal aspirates [12]. We perceived our patient was very ill and febrile and had imaging features of necrotizing pancreatitis while he hadn't any history of trauma or drug use. Also, he had hematuria without any clue of UTI or structural abnormality or any positive history. So we thought of the probability of MIS-C for him based on two organ involvement and considering the environmental factors suggesting the possibility of COVID-19 pandemic and checked inflammatory markers which became positive. His fibrinogen was high and liver aminotransferases and bilirubin were normal, so we threw down HLH or MAS diagnosis. He fulfilled RCPCH (Royal College of Pediatrics and Child Health) and CPSP (Canadian Pediatric Surveillance Program) criteria for MIS-C but his nasopharyngeal COVID-19 PCR and serology for COVID-19 IgG was negative. CDC (center for disease control) and WHO (world health organization) criteria require COVID-19 infection confirmation for MIS-C but RCPCH and CPSP do not. Radia, et al. in a systematic review of MIS-C reported that only $59 \%$ of cases were COVID-19 infection positive [14].

The American Rheumatology College recommends IVIG and steroids alone or together for MIS-C patients [12]. Our patient received $1 \mathrm{gr} / \mathrm{kg}$ IVIG and Methylprednisolone pulse in addition to supportive care, broad-spectrum antibiotics and surgical intervention but unfortunately expired after long hospitalization. We could not approve COVID-19 infection for him but it seems very likely based on epidemiology and severity and diversity of his involvement. We recommend considering the possibility of MIS-C in such unusual severe presentations in the current epidemic and start IVIG and steroid treatment even if the COVID-19 test is negative.

\section{Ethical considerations}

There was no ethical consideration to be considered in this research.

\section{References}

1. Gholami M, Arjmand R. Acute Pancreatitis Caused by COVID-19 in a Child: A Case Report. Int J Enteric Pathog. 2020; 8: 107-109.

2. Boumitri C, Brown E, Kahaleh M. Necrotizing Pancreatitis: Current Management and Therapies. Clin Endosc 2017; 50: 357-365. PubMed: https://pubmed.ncbi.nlm.nih.gov/28516758/

3. Al Mazrouei SS, Saeed GA, AI Helali AA. COVID-19-associated acute pancreatitis: a rare cause of acute abdomen. Radiol Case Rep. 2020; 15: 1601-1603.

PubMed: https://pubmed.ncbi.nlm.nih.gov/32685078/

4. de Madaria E, Capurso G. COVID-19 and acute pancreatitis:examining the causality. Nat Rev Gastroenterol Hepatol. 2021; 18: 3.

PubMed: https://pubmed.ncbi.nlm.nih.gov/33203968/

5. Arbati MM, Molseghi MH. COVID-19 Presenting as AcuteNecrotizing Pancreatitis. J Investig Med High Impact Case Rep. 2021; 9: 1-3. PubMed: https://pubmed.ncbi.nlm.nih.gov/33847153/

6. Bineshfar N, Mirahmadi A, Karbasian F, Pourbakhtyaran E, Karimi A, et al. Acute Pancreatitis as a Possible Unusual Manifestation of COVID-19 in Children. Case Rep Pediatr. 2021; 6616211. PubMed: https://pubmed.ncbi.nlm.nih.gov/33575053/

7. Gupta A, Bansal DP, Rijhwani P, Singh V. A Case Report on Acute Pancreatitis in a Patient With Coronavirus Disease 2019 (COVID-19) Pneumonia. Cureus. 2021; 13: e14628.

PubMed: https://pubmed.ncbi.nlm.nih.gov/34046267/

8. Rotar O, Khomiak I, Polanskyy O, Muskovsky Y, Railianu S, et al Case Report of Fatal Acute Necrotizing Pancreatitis in Patient with COVID-19: We Should Be Aware Of Hemorrhagic Complications. JOP. J Pancreas 2020; 21: 167-171.

9. Wifi MN, Nabil A, Awad A, Eltatawy R. COVID-induced pancreatitis: case report. Egypt J Inter Med. 2021; 33: 10.

PubMed: https://pubmed.ncbi.nlm.nih.gov/33716498/

10. AlHarmi RAR, Fateel T, Adnan JS, Al Awadhi K. Acute pancreatitis in a patient with COVID-19. BMJ Case Rep. 2021; 14: e239656.

PubMed: https://www.ncbi.nlm.nih.gov/pmc/articles/PMC7880111/

11. Nakra NA, Blumberg DA, Herrera-Guerra A, Lakshminrusimha S Multi-System Inflammatory Syndrome in Children (MIS-C) Following SARS-CoV-2 Infection: Review ofClinical Presentation, Hypothetical Pathogenesis, and Proposed Management. Children. 2020; 7: 69. PubMed: https://pubmed.ncbi.nlm.nih.gov/32630212/

12. Kiss A, Mac Daragh Ryan P, Mondal T. Management of COVID19-associated multisystem inflammatory syndrome in children: A comprehensive literature review. Prog Pediat Cardiol. 2021; 63: 101381.

PubMed: https://pubmed.ncbi.nlm.nih.gov/33850412/

13. Bugiantella W, Rondelli F, Boni M, Stella PP, Polistena A, et al. Necrotizing pancreatitis: A review of the interventions. Int J Surg. 2016; 28: S163-eS171.

PubMed: https://pubmed.ncbi.nlm.nih.gov/26708848/

14. Radia T, Williams N, Agrawal P, Harman K, Weale J, et al; Multisystem inflammatory syndrome in children \& adolescents (MIS-C): A systematic review of clinical features and presentation. Paediatr Respir Rev. 2021' 38: 51-57.

PubMed: https://pubmed.ncbi.nlm.nih.gov/32891582/ 